December 2021 (№20)

Volume 7; Issue 5; Page No. 124-144

ISSN: 2346-7916 (Print)

ISSN: 2587-5043 (Online)

\title{
Criminological Aspects of the Causes of Crime
}

\author{
Mikheil Gabunia \\ Doctor of Law, Georgian Technical University, Faculty of Law and International Relations, \\ Associate Professor \\ Lika Kavtuashvili \\ Georgian Technical University, Faculty of Law and International Relations, $4^{\text {th }}$ year student \\ Ani Aslanishvili \\ Georgian Technical University, Faculty of Law and International Relations, $4^{\text {th }}$ year student \\ Anano Motsonelidze \\ Georgian Technical University, Faculty of Law and International Relations, $4^{\text {th }}$ year student
}

\section{ARTICLE INFO}

Article History:

Received 19.10.2021

Accepted 15.11.2021

Published 20.12.2021

Keywords:

Criminology,

Criminality,

Cause

\section{ABSTRACT}

In modern criminology, the threefold division of the causes of crime is accepted: physical; Anthropological and social. However, it should be noted that in modern criminology there is an opinion that the reasons are divided into two parts. In particular, physical factors should be removed from this classification and the causes of crime should be divided into social and anthropological categories.

For modern criminology, in relation to the causes of crime, it would be most appropriate to take into account the vast experience of the past and to conduct our further research in a three-part classification: studying anthropological, physical and social causes and influencing these causes, developing prevention measures.

Experience has clearly shown that the science of criminology in the fight against crime, occupies a leading place among the social sciences, whose recommendations should be taken into account as much as possible in the social control of crime. 


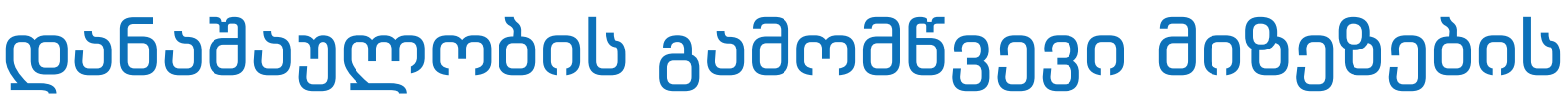

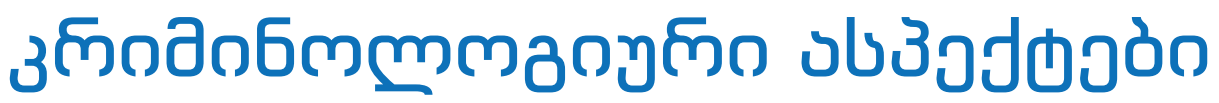

\author{
పobonm àdy6ou
}

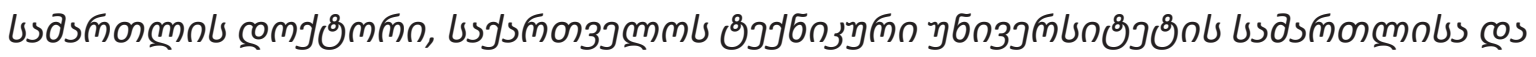

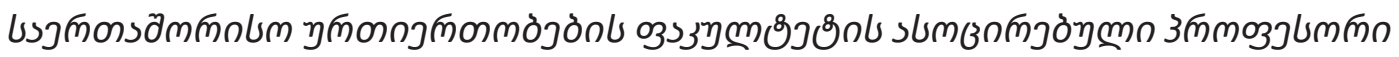

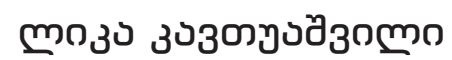

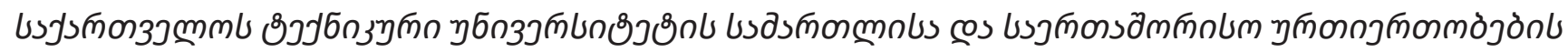

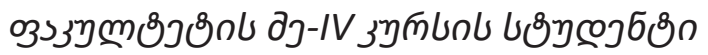

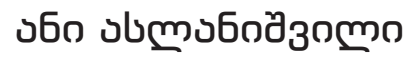

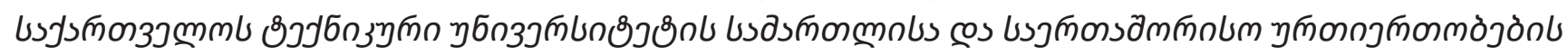

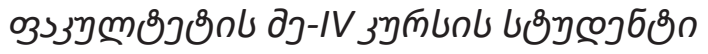

ง

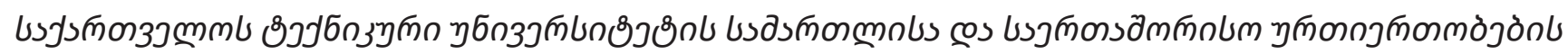

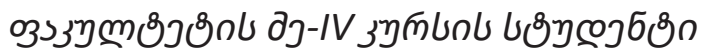

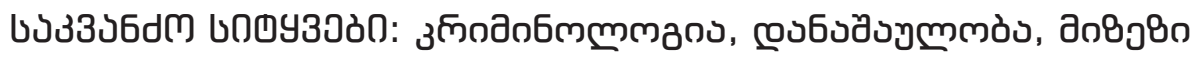

\section{ของไวงmก}

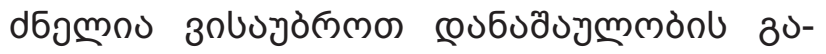

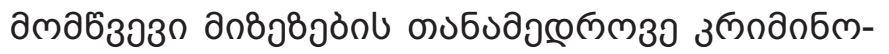

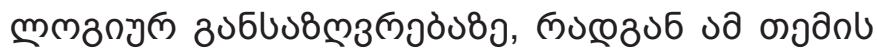

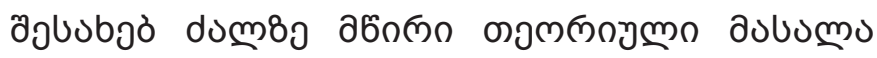

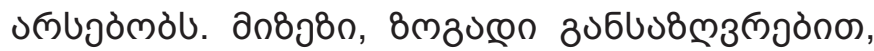

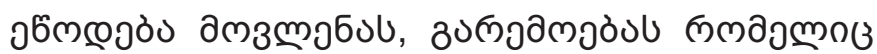

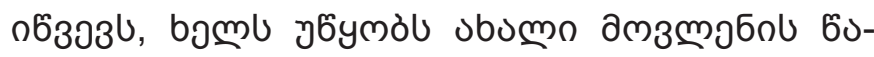

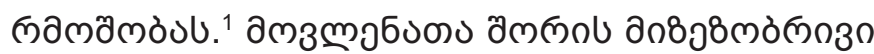

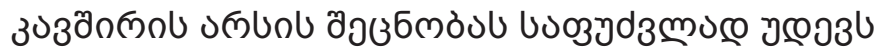

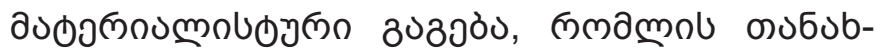

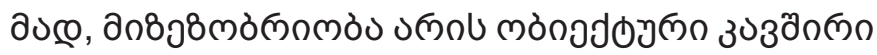

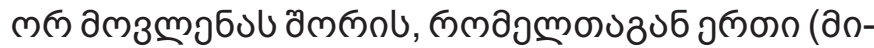

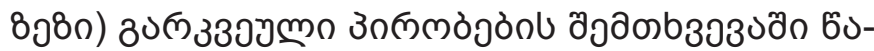

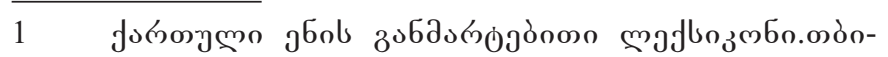
mobo. 1986. 33.297.

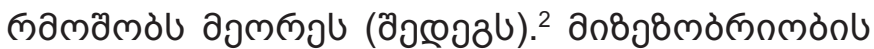

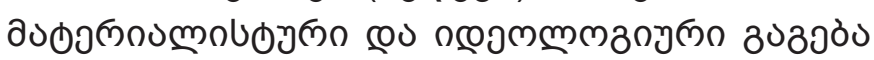

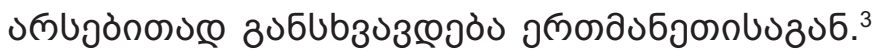

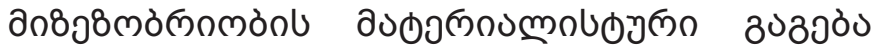

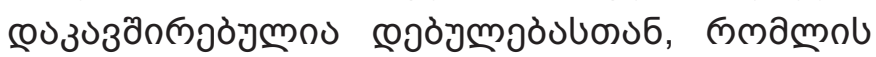

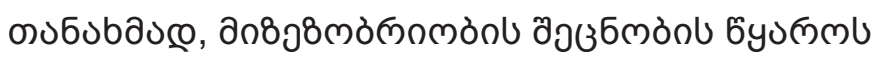

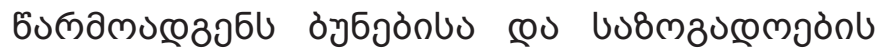

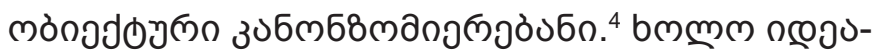

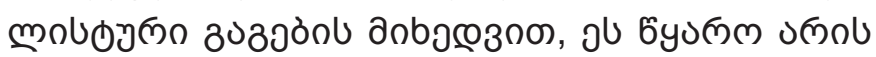

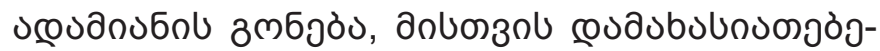

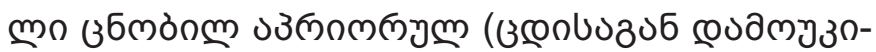

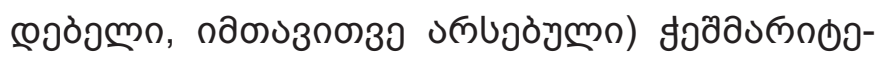

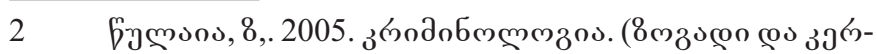

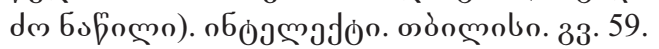
of3ว.

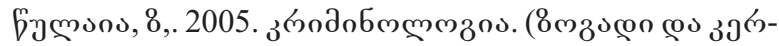

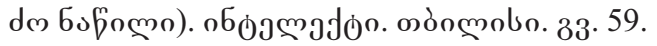




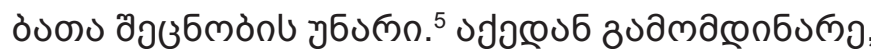

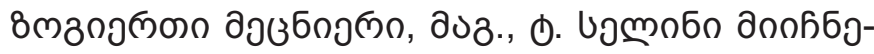

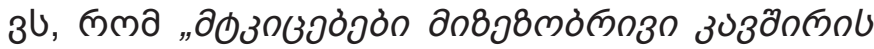

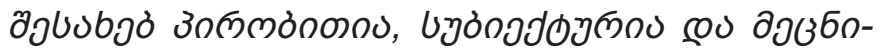

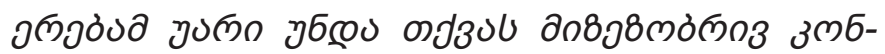

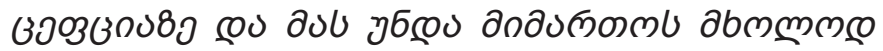

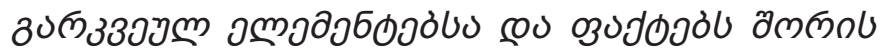

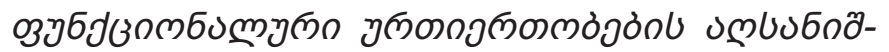

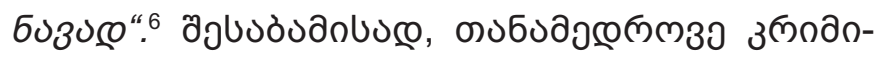

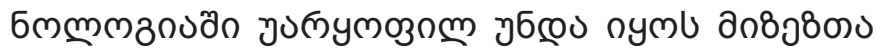

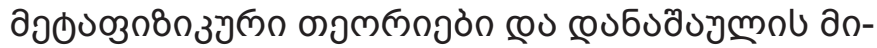

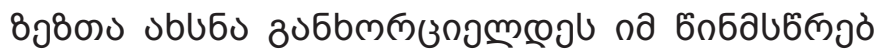

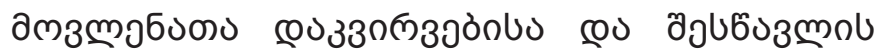

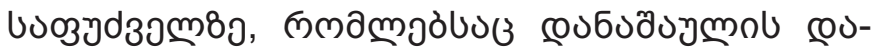

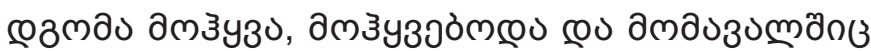

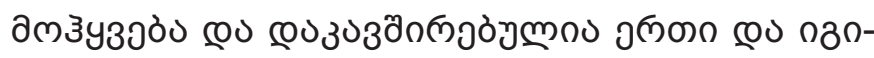

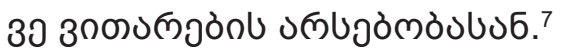

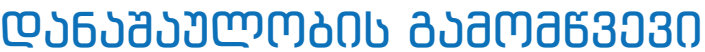

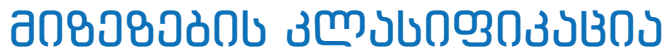

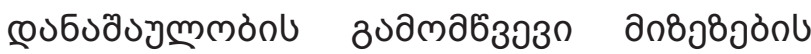

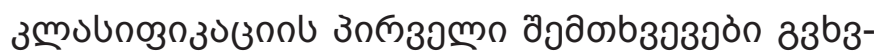

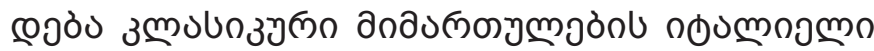

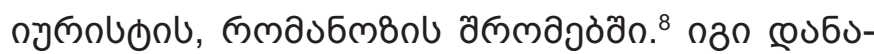

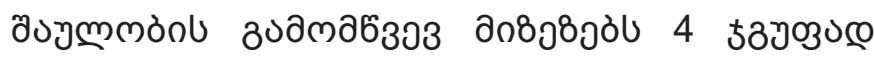
उदलभुए:

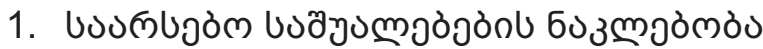

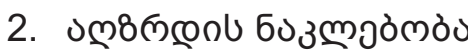

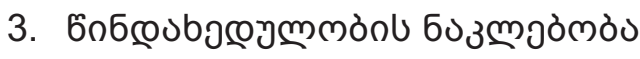

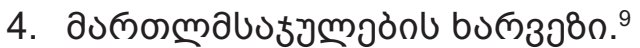

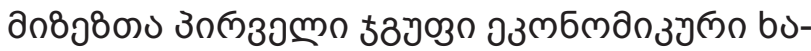

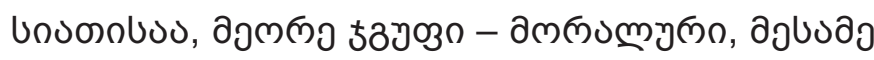

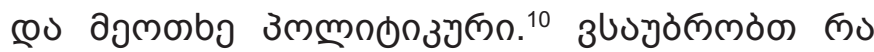

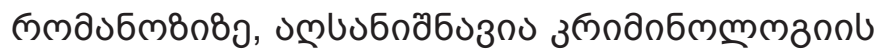

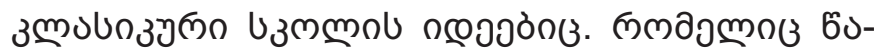

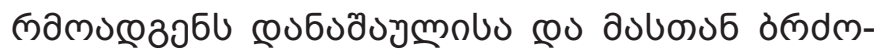
mol nূojos unbojaul, nan huamyumndœo

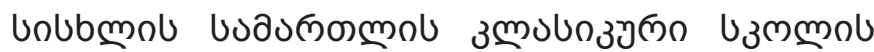

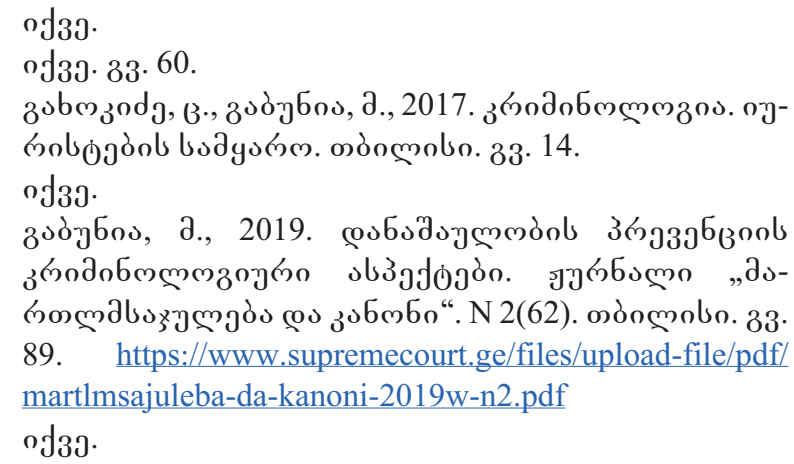

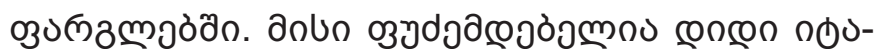

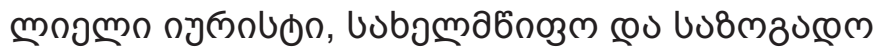

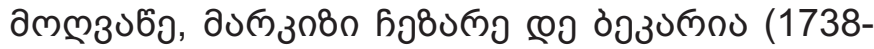

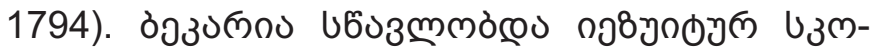

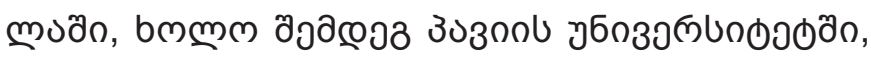

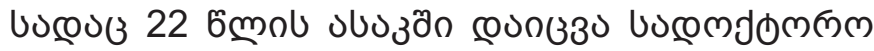

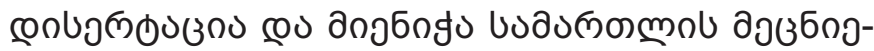

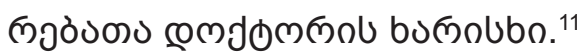

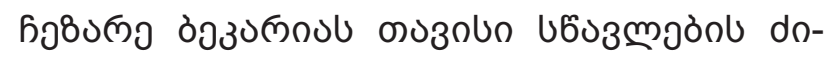

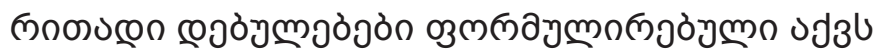

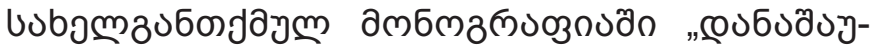

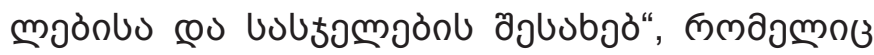

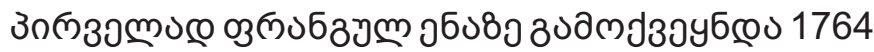

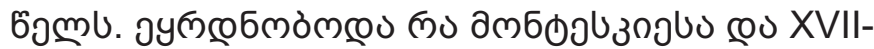

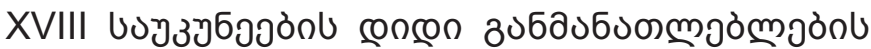

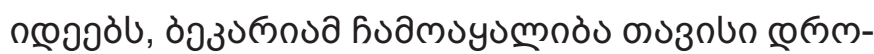

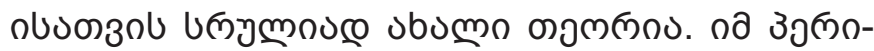

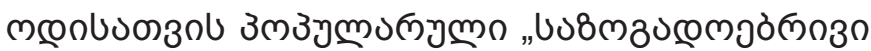

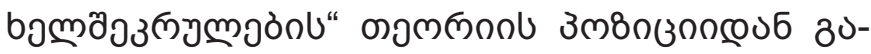

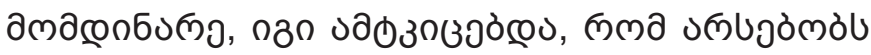

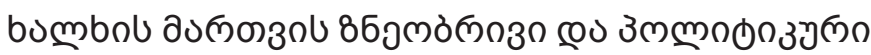

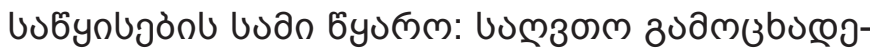

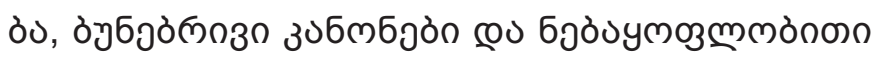

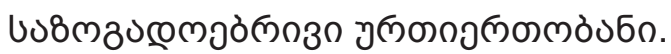

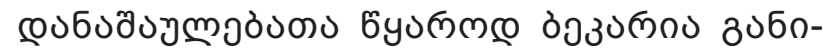

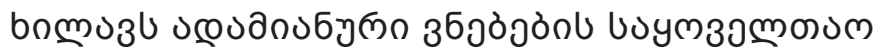

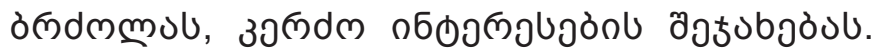

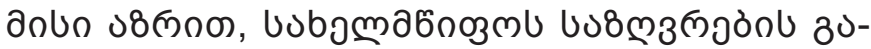

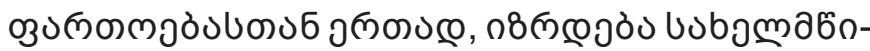

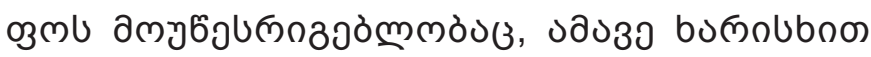

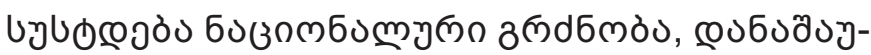

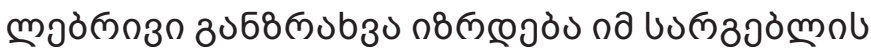

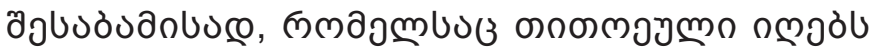

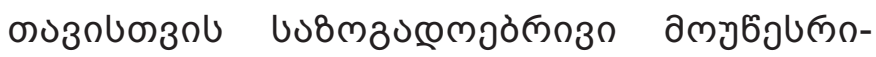

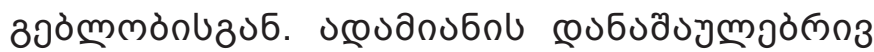

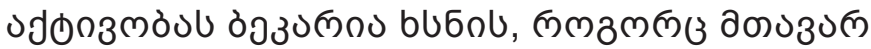

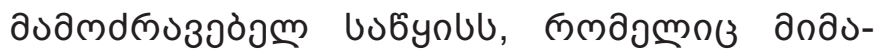

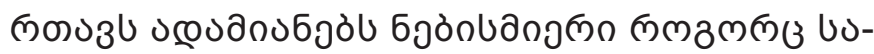

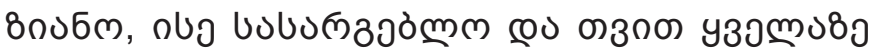

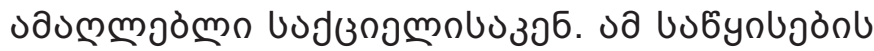

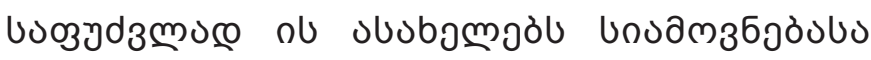

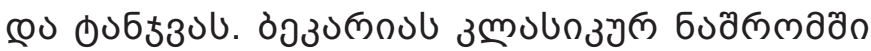

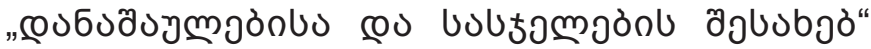

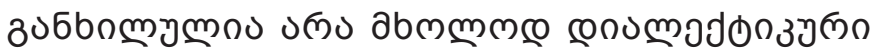

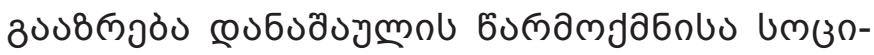

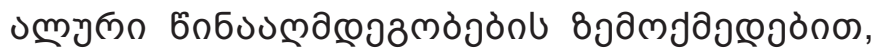

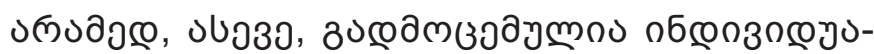

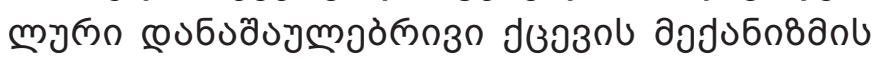

11 https://www.biography.com/scholar/cesare-beccaria 


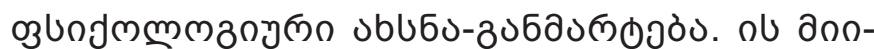

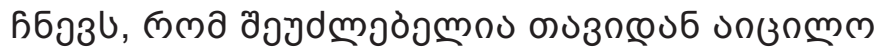

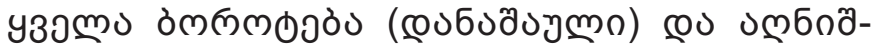

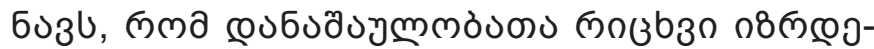

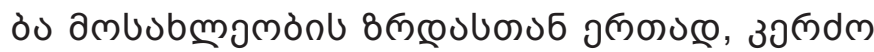

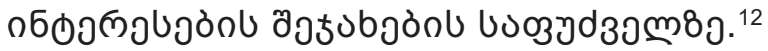

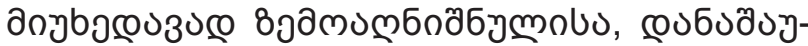

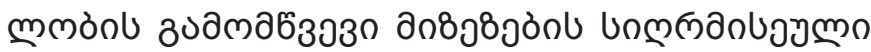

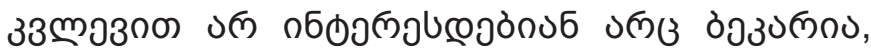

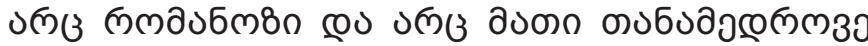

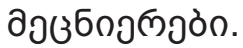

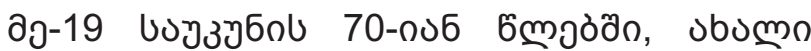

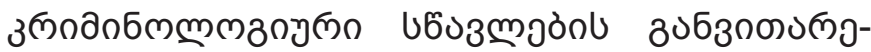

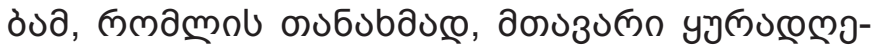

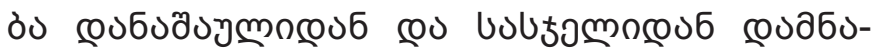

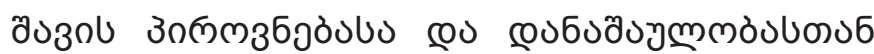

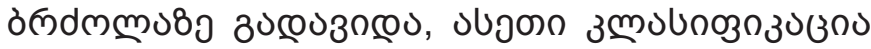

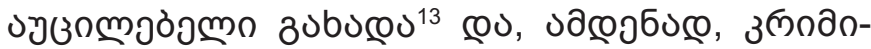

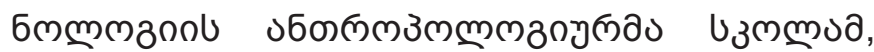

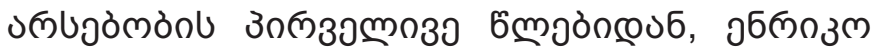

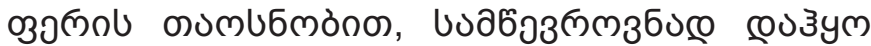

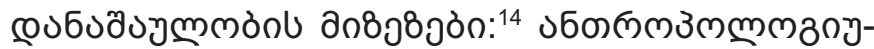

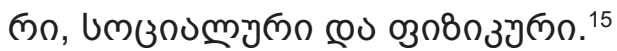

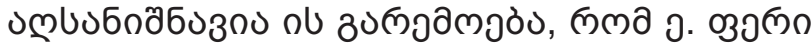

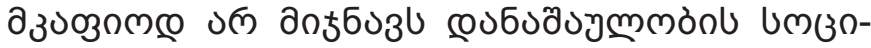

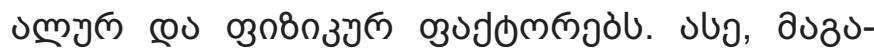

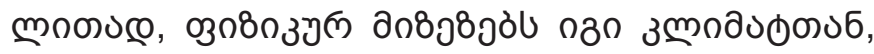

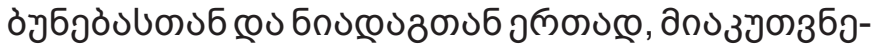

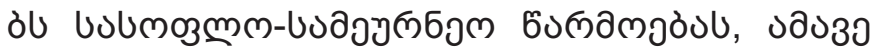

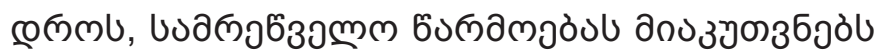

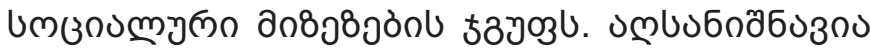

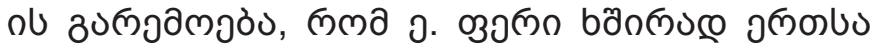

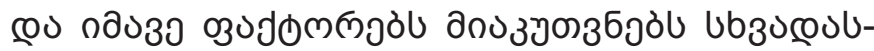

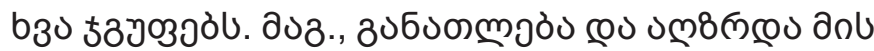

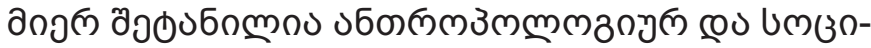

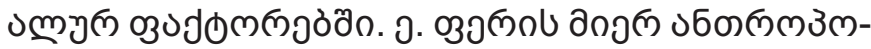

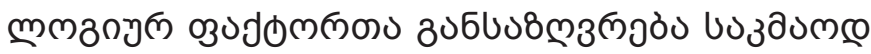

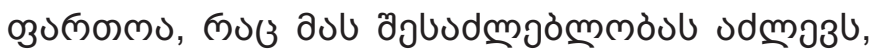

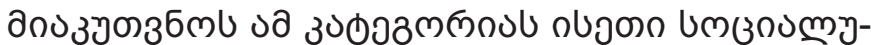

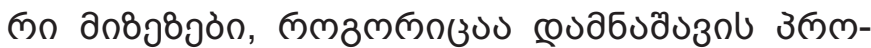

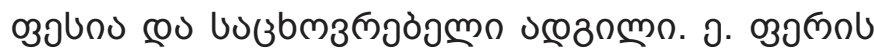

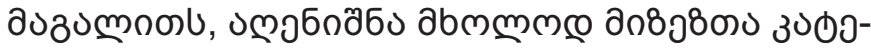

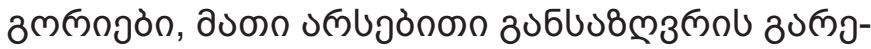

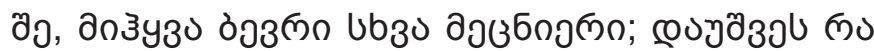

12 http://ebooks.grsu.by/criminal/3-prichiny-prestupnosti. htm

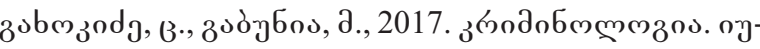

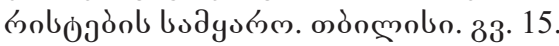
-

15 of 39

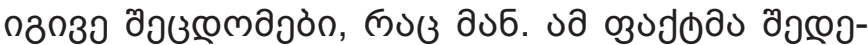

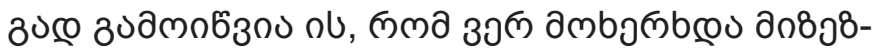

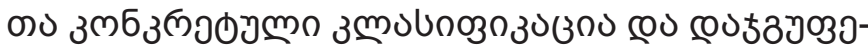

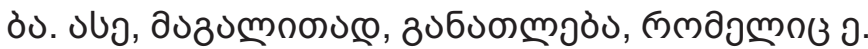

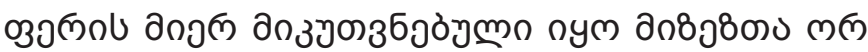

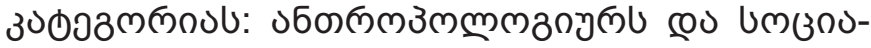

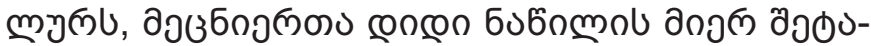

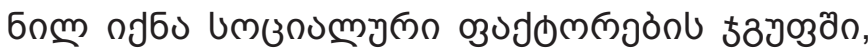

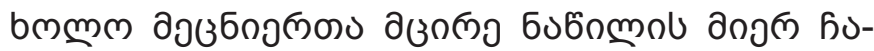

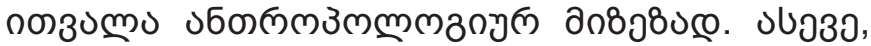

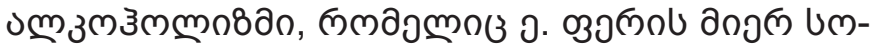

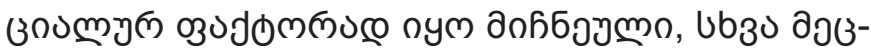

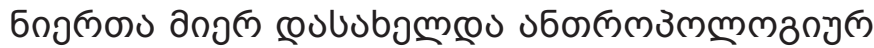

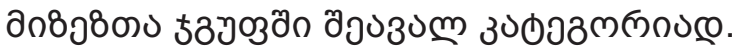

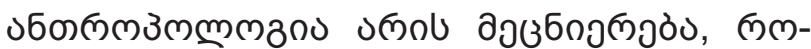

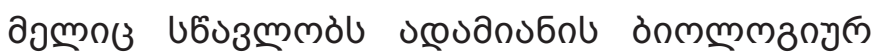

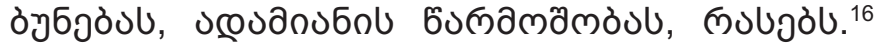

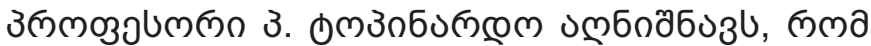

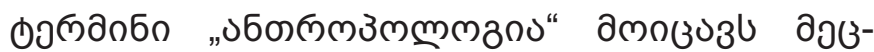

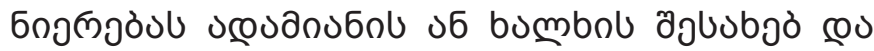

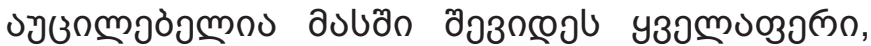

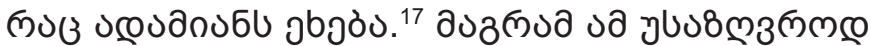

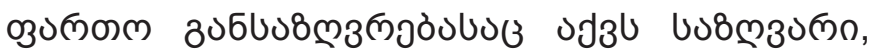

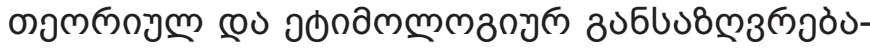

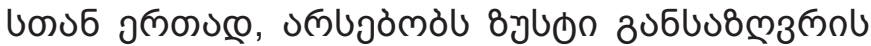

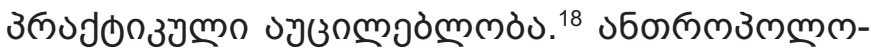

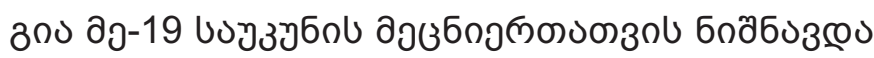

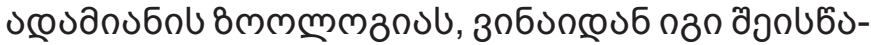

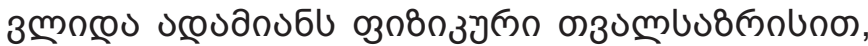

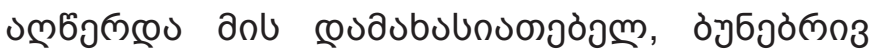

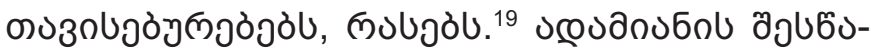

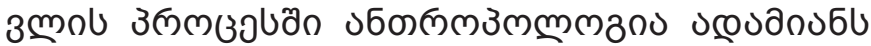

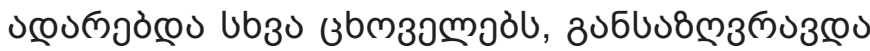

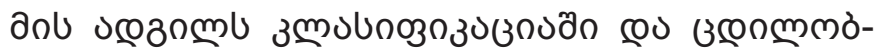

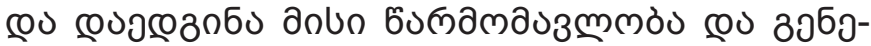

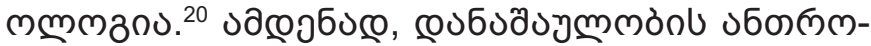

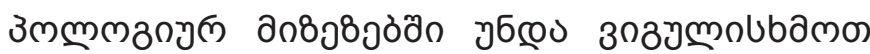

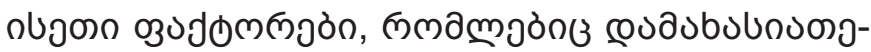

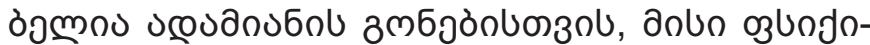

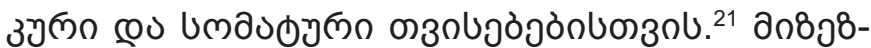

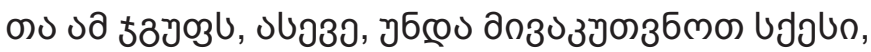

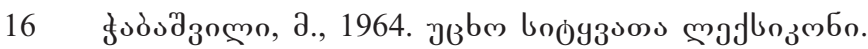
onòngolso, 33.40.

17 3

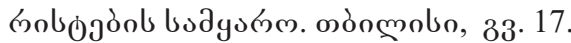

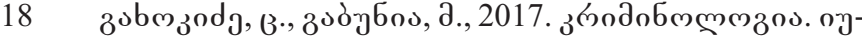

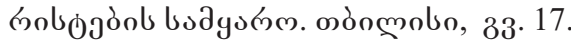

○าวง.

of39. 33.18

จงวอ.
} 


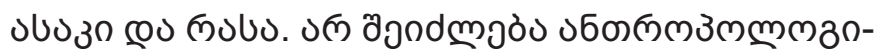

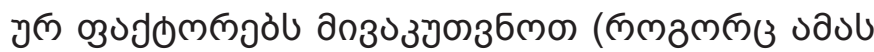

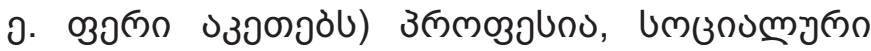

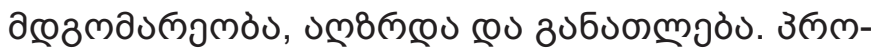

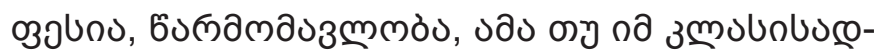

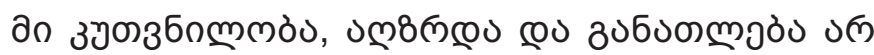

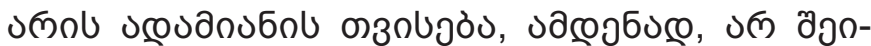

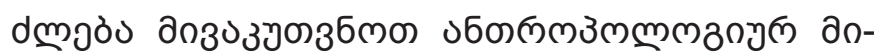

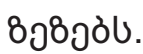

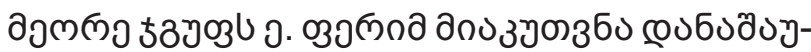

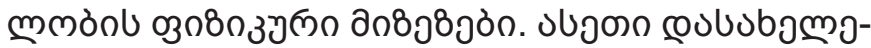

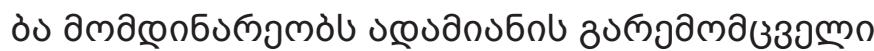

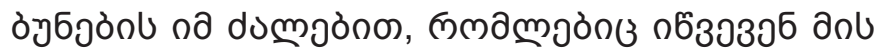

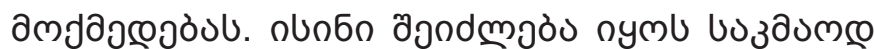

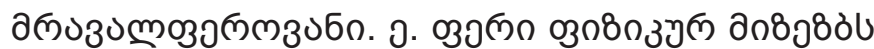

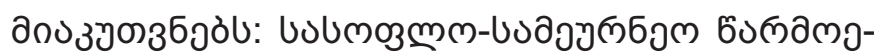

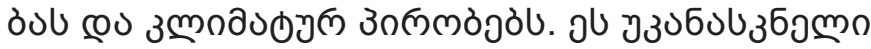

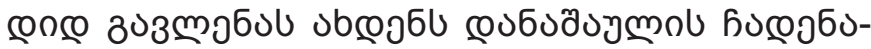

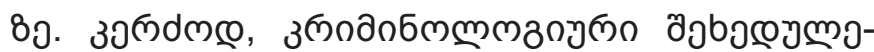

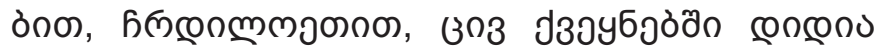

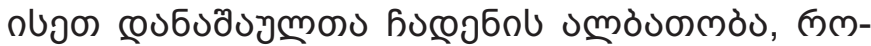

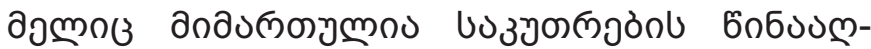

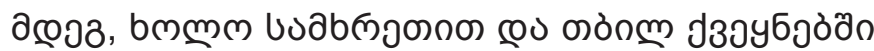

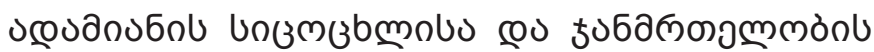

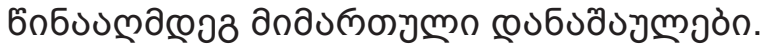

ап8э80ง a

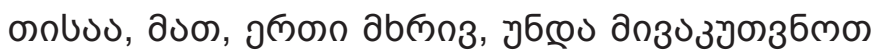

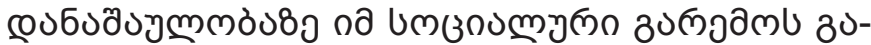

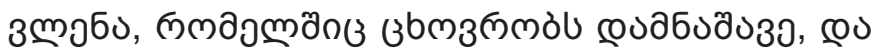

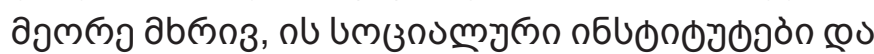

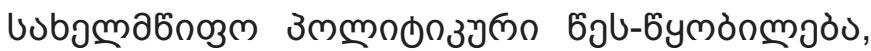

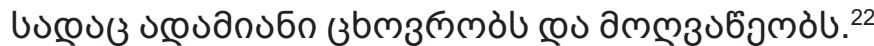

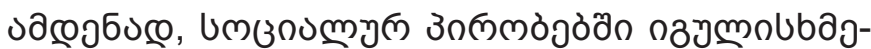

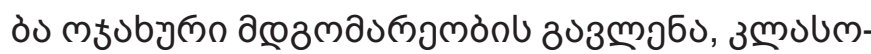

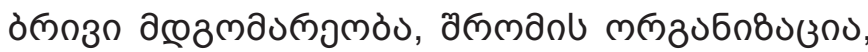

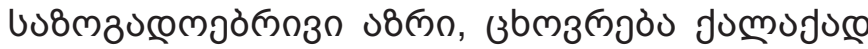

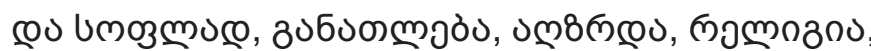

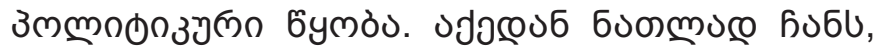

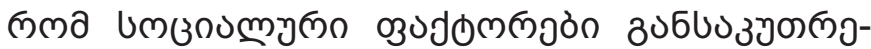

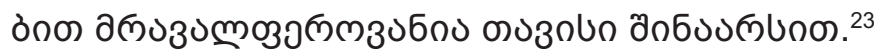

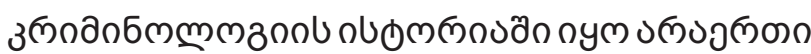

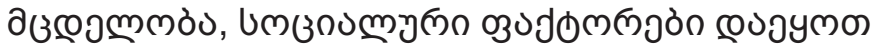

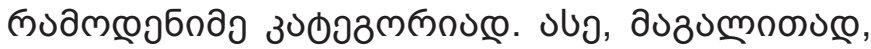

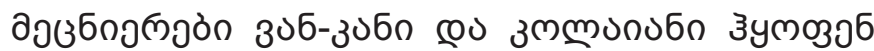

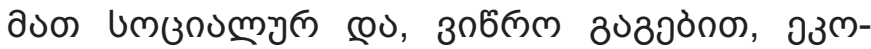

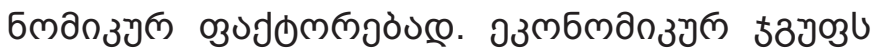

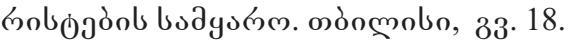

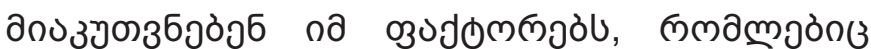

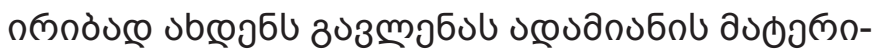

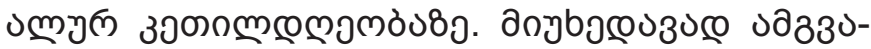

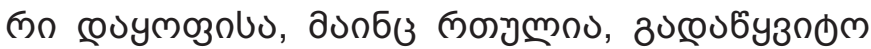

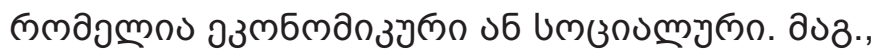

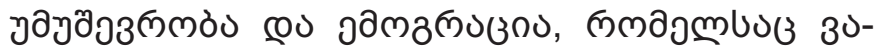

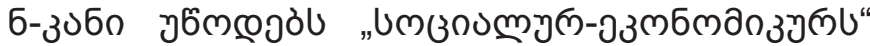

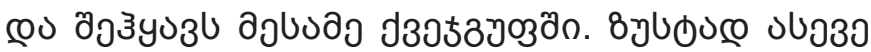

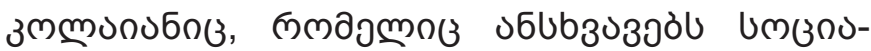

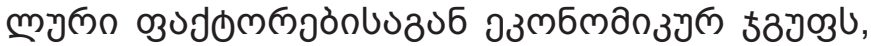

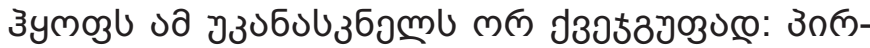

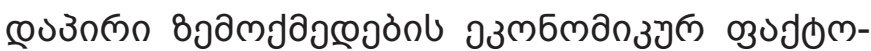

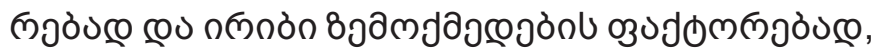

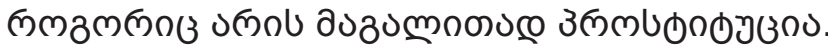

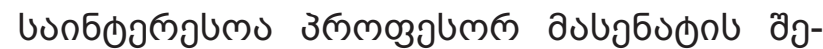
ьэкумјј

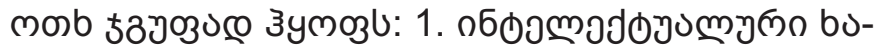

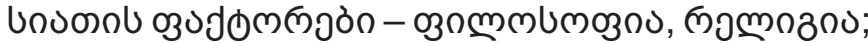

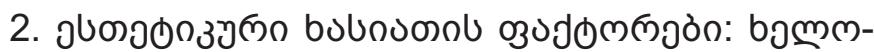

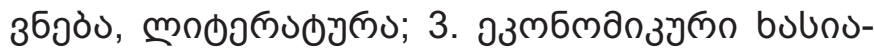

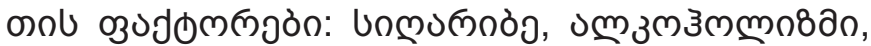

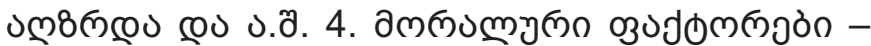

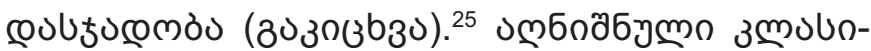

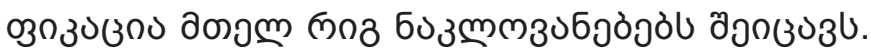

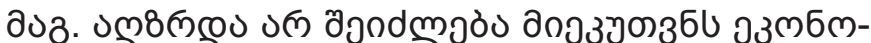

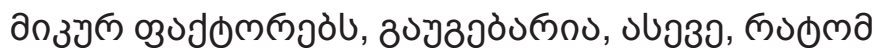

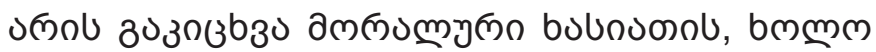

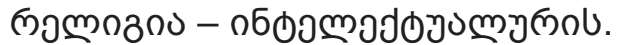

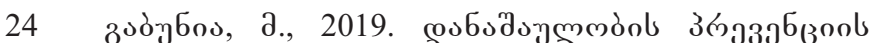

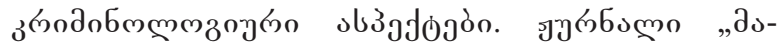

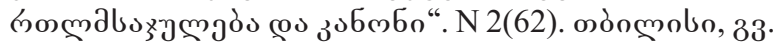
90. https://www.supremecourt.ge/files/upload-file/pdf/ martlmsajuleba-da-kanoni-2019w-n2.pdf of3ว. 


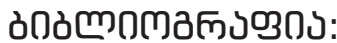

1. Ферри, Э., 1910. Уголовная социология. М.

2. Гернет, М., 1905. Социальные фракторы преступности. М.

3. Criminology. John F., Conclin. Tufts University. Ninth Edition. 2007

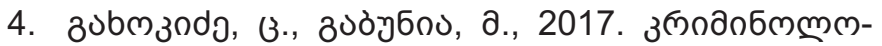

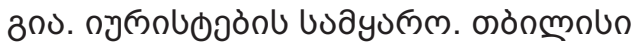

\section{BIBLIOGRAPHY:}

1. Ferri, E., 1910. Criminal sociology. M.

2. Gernet, M., 1905. Social factors of crime. M.

3. Criminology. John F., 2007. Conclin. Tufts University. Ninth Edition

4. Gakhokidze, Ts., Gabunia, M., 2017. Criminology. Publisher The world of lawyers. Tbilisi

\section{NOTES:}

1. Explanatory Dictionary of Georgian Language. Tbilisi. 1986. p.297

2. Tsulaia, Z., 2005. Criminology. (General and private part). Publisher Intellect. Tbilisi, p. 59

3. Gakhokidze, Ts., Gabunia, M., 2017. Criminology. Publisher The world of lawyers. Tbilisi, p. 14

4. Mikheil Gabunia. Criminological aspects of crime prevention. Journal "Justice and Law". N 2 (62). Tbilisi. 2019. p. 89

5. https://www.biography.com/scholar/cesare-beccaria

6. http://ebooks.grsu.by/criminal/3-prichiny-prestupnosti.htm

7. Chabashvili, M., 1964. Dictionary of foreign words. Tbilisi, p. 40 